\title{
On the Performance of the European LMDS System
}

\author{
Michel Marot ${ }^{\star}$, Monique Becker ${ }^{\star \star}$, and Paul-Vincent Marboua ${ }^{\star \star \star}$ \\ G.E.T./I.N.T.; U.M.R. 5157 S.A.M.O.V.A.R. \\ Institut National des Télécommunications \\ 9 rue Charles Fourier \\ 91011 EVRY-FRANCE \\ Phone : (33) 1-60-76-47-81 \\ Fax : (33) 1-60-76-47-80 \\ \{Michel.Marot, Monique.Becker, Paul-Vincent. Marboua\}@int-evry.fr
}

\begin{abstract}
This paper investigates the performance of the European LMDS system. The collision rate and the Laplace transform of the access delay to the shared medium, are analytically computed and used to derive numerically the mean of the response time (the access delay to the shared medium). At last, the performance of the LMDS system when the contention mode is used for dynamic reservation request is analytically studied.

Among several results, we prove that the use of the max parameter in the LMDS collision recovery mechanism prevents the system from unstability, whatever the collision rate is. It differs from results derived in other papers for binary exponential backoff algorithms without max parameter. It appears also that stability has to be carefully defined. Several definitions of stability may be chosen and the derived conclusions are then different.
\end{abstract}

Keywords: Contention access mode, fixed point, exponential backoff, collision rate, analytical study

\section{Introduction}

Local Multipoint Delivery Systems (LMDS [2]) is designed to be used to implement interactive services in a DVB environment, providing a bi-directional communication path between the user terminal and the service provider. A DVB downstream channel is broadcasting video, audio and data from a base station to clients. LMDS can be used to provide wireless access from the user to the base station, for instance to provide Internet access. Data are sent downstream from the base station to the clients in a FIFO manner, each client looking at the downstram traffic to see whether he is concerned with the received packets

\footnotetext{
* Michel Marot is an Assistant Professor at I.N.T.

$\star \star$ Monique Becker is a Professor at I.N.T.

$\star \star *$ Paul-Vincent Marboua is a Master student at I.N.T.
} 
or not. The upstream channel is a slotted TDMA multiple access channel. It can be shared in a fixed mode, where each client has a dedicated sequence of slots, in a contention mode, or in a dynamical mode where clients wanting to send data send reservation requests to the base station which temporarily reserves data slots to the user. The reservation requests can be sent in contention mode. Contention mode, which use a collision recovery mechanism similar to the standard Binary Backoff Exponential (BEB) mechanism used in ethernet [8] or wireless LAN [9] standards, and mixed contention reservation modes are difficult to analyse and are the subject of this paper.

For thirty years, there has been a lot of studies about BEB (e.g. [1], [3]). An excellent survey on this area is given in [4]. The authors reference several papers which have proved that BEB is unstable for an infinite node model while it is stable for a finite node model, if the system arrival rate is small enough but unstable if the arrival rate is too large. Of course, when the throughput of each of the sources increases, or for a given throughput when the number of sources increases, the expected number of packets waiting to be transmitted or the expected delay before transmission increases. Of course LMDS is unstable in that way. But for a given number of users and a given throughput of the requests of each of them, in case the throughput of the successfully transmitted packets tends to zero when the requests throughputs is increasing, the network will be said to be unstable and stable otherwise In [4], an interesting new approach is proposed where the collision rate is numerically derived as the solution of a problem of fixed point. Actually, the collision rate depends on the emission rate of the sources but the emission rate also depends on the collision rate: there is an equation to invert. The authors also study the stability of the BEB. They prove that if the collision rate is larger than 0.5 the system is unstable.

In the present paper we study the collision rate as a fixed point, but not only in saturation condition as the authors of [4] do and we propose a method to analytically analyze the variations of the fixed point of the collision rate as a function of the parameters of the system (section 2). We prove that with max and min values bounding backoff exponent, the LMDS system can be stable in some cases even when the collision rate is greater than 0.5 . Moreover, we also derive (in section 3) the Laplace transform of the access delay to the share medium which permits us to numerically obtain the moments of the service and response times. At last (section 4), we consider the mixed scenario where data are sent with dynamical reservation mechanism, the dynamical reservation requests being sent through the contention mode. The distribution of the reservation queue on the base station and the total mean response time are analytically derived. We show that there is an optimal value for the amount of contention slots compared to the amount of data slots in this scenario, which is a tradeoff between the collision rate and the amount of the data slots.

\section{The Collision Rate}

The system is constituted by $N$ clients sending ATM cells to queues implementing the MAC protocol. Each client has such a single queue. Each of these queues 
sends traffic through the upstream shared channel to the base station. The time is divided in periods of $3 \mathrm{~ms}$ and each period is divided in $m=18$ ATM slots. A queue can send at most one cell per period. When it just sends a cell, it waits during three periods for an acknowledgment to know if a collision occured or if the cell has been successfully transmitted. Actually, it is possible that simultaneous transmissions occur in a single slot, which is called a collision. If a collision has occured, the packet may be retransmitted. A counter records the number of collisions encountered by the packet and generates a backoff_exponent which is included between a min and a max value.The retransmission will be scheduled in a slot whose number is uniformly distributed between 1 and $2^{\text {backoff_exponent }}$. The backoff_exponent is encreased by one when a new collision happens, but when it reaches its max value. If the counter reaches the maximum number, it remains at this value regardless of the number of subsequent collisions.

For clarity of the description, let us call applications the clients generating traffic, which are modeled as Poisson processes, and sources the queues below the applications and above the shared medium. All applications are assumed to be Poisson with identical rate. Let us define the following notations:

$-\lambda$ : the rate of each Poisson source

$-N$ : the number of sources

- $U$ : the utilisation factor of the sources

- $A_{c k}$ : number of periods a source waits to receive the acknowledgement.

- min, max: parameters of the LMDS collision resolution algorithm

$-m$ : number of slots per period

$-n_{r}$ : number of retransmissions for a given cell to be transmitted successfully

- $X$ : random variable representing the time in periods necessary for a source to transmit successfully a given cell (the source service time)

- $Y$ : random variable representing the time in periods between two transmissions, whatever they are successful or not (i.e.: with collision), when the queue of the source is not empty

$-p_{c}$ : the collision rate

$-p_{e}$ : effective emission rate of the sources ( $p_{e} \geq \lambda$ because of retransmissions).

$Z \hookrightarrow$ Uniform $(\llbracket A ; B \rrbracket)$ means that the random variable $\mathrm{Z}$ is discrete and uniformly distributed between $\mathrm{A}$ and $\mathrm{B}$ and $\lceil r\rceil$ denotes the upper integer part.

\subsection{The Equation of the Collision Rate}

In each period slot, the collision rate depends on the emission rate $p_{e}$ of the sources. It depends also of the number of emitting sources $k$ (see [6] for details):

$$
p_{c}=1-\left(1-\frac{1}{m} p_{e}\right)^{N-1}
$$

Moreover, $p_{e}=\frac{U}{\mathbb{E}[Y]}$ and, each source being an $\mathrm{M} / \mathrm{G} / 1$ queue, $U=\rho=\lambda \mathbb{E}[X]$. We may determine $\mathbb{E}[X]$ and $\mathbb{E}[Y]$. 
First approximation: Theoretically, when a collision occurs, the source has to wait for a given number of slots before retransmitting. Because we choose the period as time unit, and because this number of slots is generated according to a discrete uniform distribution between 1 and $2^{\text {min }}, 2^{i}$ or $2^{\text {max }}$, we consider that the corresponding time to wait before retransmitting is generated according to a discrete uniform distribution between 1 and $\left\lceil\frac{2^{m i n}}{m}\right\rceil, 1$ and $\left\lceil\frac{2^{j}}{m}\right\rceil$ or 1 and $\left\lceil\frac{2^{\max }}{m}\right\rceil$ (it is an approximation).

The number of periods necessary to transmit successfully a cell is:

$$
X=A_{c k}\left(1+n_{r}\right)+\sum_{j=1}^{n_{r}} i_{j}
$$

where,

$$
\begin{aligned}
& i_{j} \hookrightarrow \text { Uniform }\left(\llbracket 1 ;\left[\frac{2^{\text {min }}}{m}\right\rceil \rrbracket\right) \text { for } 1 \leq i_{j} \leq \min \\
& i_{j} \hookrightarrow \text { Uniform }\left(\llbracket 1 ; \frac{2^{j}}{m} \rrbracket \rrbracket\right) \text { for } \min \leq i_{j} \leq \max \\
& i_{j} \hookrightarrow \text { Uniform }\left(\llbracket 1 ;\left\lceil\frac{2^{\max }}{m}\right\rceil \rrbracket\right) \text { for } \max \leq i_{j}
\end{aligned}
$$

If $P\left(n_{r}\right)$ denotes the probability that the number of retransmissions is $n_{r}$,

$$
\mathbb{E}[X]=A_{c k}+A_{c k} \mathbb{E}\left[n_{r}\right]+\sum_{n_{r}=1}^{+\infty} P\left(n_{r}\right) \mathbb{E}\left[\sum_{j=1}^{n_{r}} i_{j} / n_{r}\right]
$$

Since $n_{r}$ is geometrically distributed $P\left(n_{r}\right)=p_{c}^{n_{r}}\left(1-p_{c}\right), \mathbb{E}\left[n_{r}\right]=\frac{p_{c}}{1-p_{c}}$ and

$$
\begin{aligned}
& \sum_{n_{r}=1}^{+\infty} P\left(n_{r}\right) \mathbb{E}\left[\sum_{j=1}^{n_{r}} i_{j} / n_{r}\right]=\sum_{n_{r}=1}^{m i n}\left[\left(1-p_{c}\right) p_{c}^{n_{r}} \sum_{j=1}^{n_{r}} \frac{1}{2}\left(\left\lceil\frac{2^{m i n}}{m}\right\rceil+1\right)\right] \\
& +\sum_{n_{r}=m i n+1}^{m a x}\left(1-p_{c}\right) p_{c}^{n_{r}}\left[\sum_{j=1}^{m i n} \frac{1}{2}\left(\left\lceil\frac{2^{m i n}}{m}\right\rceil+1\right)+\sum_{j=m i n+1}^{n_{r}} \frac{1}{2}\left(\left\lceil\frac{2^{j}}{m}\right\rceil+1\right)\right] \\
& +\sum_{n_{r}=m a x+1}^{+\infty}\left(1-p_{c}\right) p_{c}^{n_{r}}\left[\sum_{j=1}^{m i n} \frac{1}{2}\left(\left\lceil\frac{2^{m i n}}{m}\right\rceil+1\right)+\sum_{j=m i n+1}^{m a x} \frac{1}{2}\left(\left\lceil\frac{2^{j}}{m}\right\rceil+1\right)\right. \\
& \left.+\sum_{n_{r}=m a x+1}^{n_{r}} \frac{1}{2}\left(\left\lceil\frac{2^{m a x}}{m}\right\rceil+1\right)\right]
\end{aligned}
$$

Second approximation: by approximating $\left\lceil\frac{2^{j}}{m}\right\rceil$ by $\frac{2^{j}}{m}$, the expression (4) can be simplified in

$$
\mathbb{E}[X]=\frac{1}{1-p_{c}}\left[A_{c k}+\frac{1}{2} p_{c}\left(\frac{2^{m i n}}{m}+1\right)+\frac{\left(\left(2 p_{c}\right)^{m i n+1}-\left(2 p_{c}\right)^{m a x+1}\right)}{4 m\left(1-2 p_{c}\right)}\right]
$$


Similarly, the expectation of the time between two transmissions of a source when it is not empty is

$$
\begin{aligned}
\mathbb{E}[Y] & =\mathbb{E}\left[A_{c k}+i_{n_{r}}\right]=\sum_{n_{r}=1}^{+\infty} \mathbb{E}\left[A_{c k}+i_{n_{r}} / n_{r}\right] P\left(n_{r}\right)+A_{c k} P(0) \\
& =\left[A_{c k}+\frac{1}{2} p_{c}\left(\frac{2^{\text {min }}}{m}+1\right)+\frac{\left(\left(2 p_{c}\right)^{m i n+1}-\left(2 p_{c}\right)^{\text {max }+1}\right)}{4 m\left(1-2 p_{c}\right)}\right]
\end{aligned}
$$

So, the effective emission probability (probability of a cell emission including retransmission) is:

$$
p_{e}=\lambda \frac{\mathbb{E}[X]}{\mathbb{E}[Y]}=\frac{\lambda}{1-p_{c}}
$$

And the collision probability becomes:

$$
p_{c}=1-\left(1-\frac{1}{m} \frac{\lambda}{1-p_{c}}\right)^{N-1}
$$

This result is similar to the one found in [4], except that in our case the sources are not saturated but are fed by Poisson processes. It can be noticed that, contrary to the results found in [4], due to the existence of the max parameter, the infinite sums in (4) and (6) always converge, even when $p_{c} \geq \frac{1}{2}$. There are no more stability problem. The drawback will be that there can be less sources in our study than in [4].

\subsection{The Collision Rate as a Function of $\lambda$}

We may study the variations of $p_{c}$ as a function of $\lambda$. The collision rate can be found by inverting the equation (8). A possible parametrisation is:

$$
\left\{\begin{array}{l}
p_{c}=1-t^{N-1} \\
\lambda=m t^{N-1}(1-t)
\end{array}\right.
$$

The variations of $p_{c}$ and $\lambda$ are given in Fig. 3 and Fig. 4 (for $m=18$ and $A_{c k}=3$ ).

These curves represent all the solutions of equation 8 . The interesting part of this curve is the portion increasing from 0 to $1-\left(\frac{N-1}{N}\right)^{N-1}$ when $\lambda$ increases from 0 to $\frac{m}{N}\left(\frac{N-1}{N}\right)^{N-1}$ if it is less than 1 or to 1 otherwise. The other parts are due to the fact that equation (8) is derived with a sequence of implications and not with a sequence of equivalences, so $p_{c}$ is solution of $(8)$, but all solutions of (8) are not necessarely $p_{c}$.

When $N$ is enough large, the maximum value of the collision rate is 1 $\left(\frac{N-1}{N}\right)^{N-1}$ and is obtained for the maximum possible value of the application 


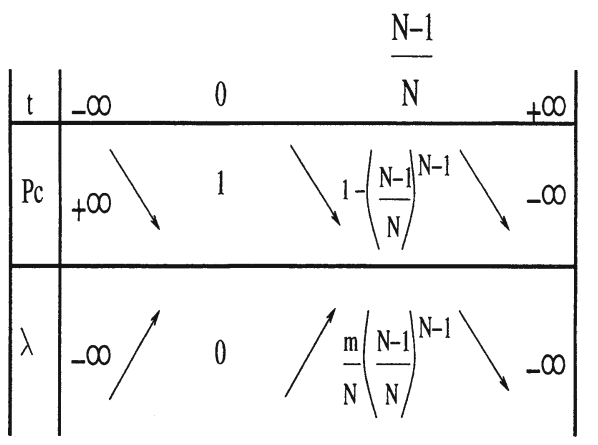

Fig. 1. $p_{c}$ and $\lambda$ variations when $\mathrm{N}$ is even

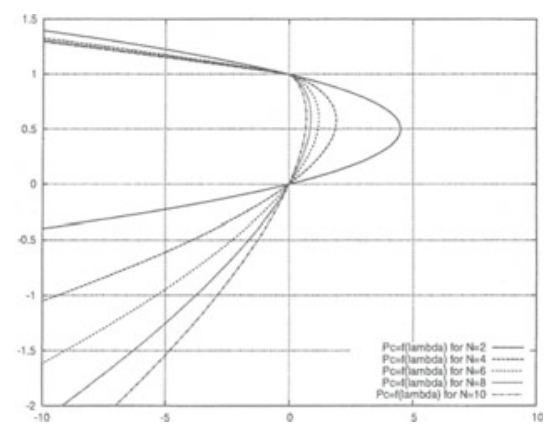

Fig. 3. $p_{c}$ as a function of $\lambda$ when $\mathrm{N}$ is even

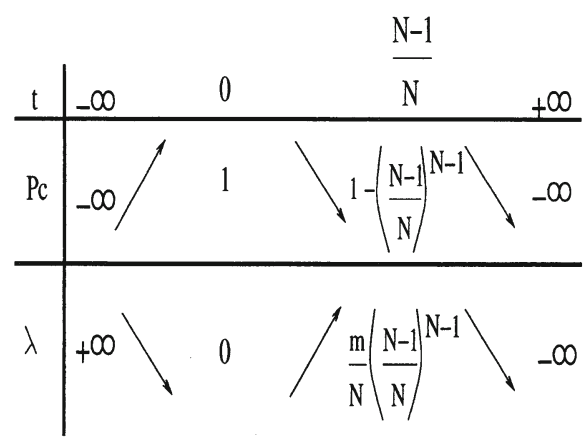

Fig. 2. $p_{c}$ and $\lambda$ variations when $\mathrm{N}$ is odd

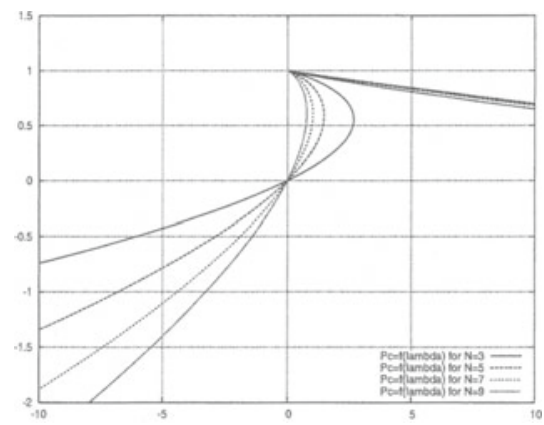

Fig. 4. $p_{c}$ as a function of $\lambda$ when $\mathrm{N}$ is odd

rate $\lambda_{\max }=\frac{m}{N}\left(\frac{N-1}{N}\right)^{N-1}$. The maximum happens when $N \frac{\lambda_{\max }}{1-p_{c_{\max }}}=m$, i.e. when the shared medium is saturated (the product of the total throughput by the expected value of the number of transmissions and by the mean service time is one). Due to the fact that a source can send at most one cell per period, this case may only appear when there is a sufficiently large number of sources $N$. In this case,

$$
\lim _{N \rightarrow+\infty} p_{c}=1-\frac{1}{e}
$$

Contrary to what stands in [4], the maximum value of the collision rate is $1-\frac{1}{e}$ and thus is greater that 0.5 ; it is explained by the use of the max parameter of the collision recovery algorithm.

$p_{c}$, derived by simulation, is plotted in Fig. 6 as a function of $\lambda$ for different number of sources. Results are obtained with confidence intervals less or equal to $5 \%$. The curves have been truncated at a value of $\lambda$ corresponding to he 
maximal possible load of the queues upstream the shared channel. There is an excellent match between simulations and analytical results.

\subsection{The Collision Rate as a Function of $N$}

The maximum possible rate per application is $\lambda_{\max }=\frac{1}{\mathbb{E}[X]}$, otherwise queues are saturated. The study of $p_{c}$ as a function of $N$ when all applications send traffic at their maximum possible rate is derived from the following expression:

$$
p_{c}=1-\left(1-\frac{1}{m} \frac{1}{A_{c k}+\frac{1}{2} p_{c}\left(\frac{2^{m i n}}{m}+1\right)+\frac{\left(\left(2 p_{c}\right)^{m i n+1}-\left(2 p_{c}\right)^{m a x+1}\right)}{4 m\left(1-2 p_{c}\right)}}\right)^{N-1}
$$

When $\mathrm{N}$ increases, $p_{c}$ increases and so the service time of the sources, that is the time for a source to successfully transmit a cell on the medium to the base station, increases too. For the queues of the sources to be stable, the applications may decrease accordingly their traffic generation rate.

When $\mathrm{N}$ reaches an enough large $N_{\text {lim }}$ value, the maximum collision rate is reached because the maximum capacity of the shared channel is reached and the applications have to decrease their rate hyperbolically according to the expression $\lambda_{\max }=\frac{m}{N}\left(\frac{N-1}{N}\right)^{N-1} \cdot N_{\text {lim }}$ is the solution of the following equation:

$$
\frac{1}{\mathbb{E}[X]}=\frac{m}{N}\left(\frac{N-1}{N}\right)^{N-1}
$$

with $p_{c}=1-\left(\frac{N-1}{N}\right)^{N-1}$. When $N$ is large, (12) can be solved with a limited expansion. The solution is:

$$
\begin{aligned}
N_{l i m} & =m\left[A_{c k}+\frac{1}{2}\left(1+\frac{2^{m i n}}{m}\right)\left(1-\frac{1}{e}\right)+\frac{\left(2-\frac{2}{e}\right)^{m i n+1}-\left(2-\frac{2}{e}\right)^{\text {max }+1}}{4 m\left(\frac{2}{e}-1\right)}\right] \\
& +\frac{1}{A_{c k}+\frac{1}{2}\left(\frac{2^{m i n}}{m}+1\right)\left(1-\frac{1}{e}\right)+\frac{1}{4 m\left(\frac{2}{e}-1\right)}\left(\left(2-\frac{2}{e}\right)^{m i n+1}-\left(2-\frac{2}{e}\right)^{m a x+1}\right)} \\
& \times\left[\frac{1}{2 e}\left(1+\frac{2^{m i n}}{m}\right)-\frac{1}{4 m\left(\frac{2}{e}-1\right)} \times\left[( 2 - \frac { 2 } { e } ) ^ { m i n + 1 } \left(\frac{2(m i n+1)}{2 e-2}\right.\right.\right. \\
& \left.\left.\left.+\frac{2}{2-e}\right)-\left(2-\frac{2}{e}\right)^{m a x+1}\left(\frac{2(\max +1)}{2 e-2}+\frac{2}{2-e}\right)\right]\right]
\end{aligned}
$$

In figure 5 the maximal value of $\lambda$ is plotted as a function of $\mathrm{N}$, for different values of max, and the corresponding collision rate. The limit values of $p_{c}(1-$ $\left(\frac{N-1}{N}\right)^{N-1}$ ) and of $\lambda\left(\frac{m}{N}\left(\frac{N-1}{N}\right)^{N-1}\right)$ are also plotted. For $\mathrm{N}$ around 70 , which corresponds to $N_{\text {lim }}, \lambda_{\max }$ and $p_{c_{\max }}$ reach their limit values. For $N>N_{\text {lim }}$, those curves may not be considered but instead their limit values.

It can be noticed that the influence of the $\max$ parameter is very marginal. 


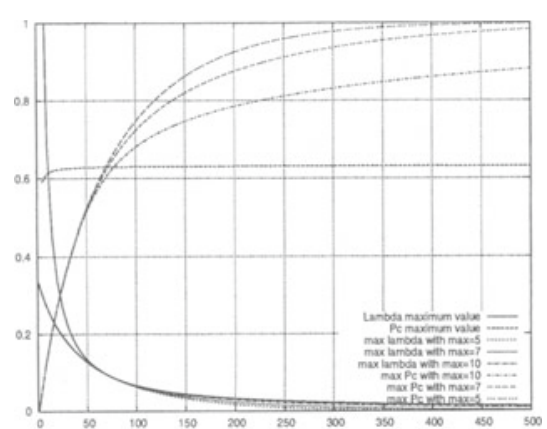

Fig. 5. $\lambda_{\max }$ and $p_{c}$ as a function of $N$, for different values of $\max$

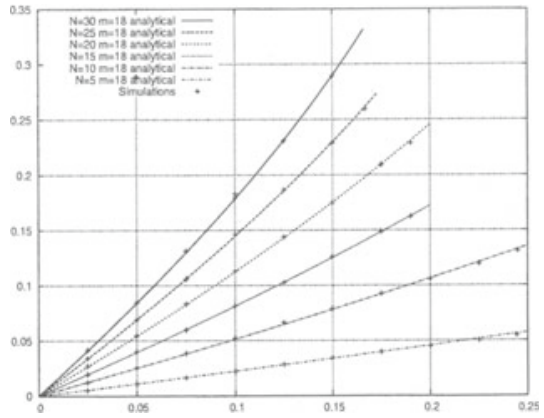

Fig. 6. Comparison between simulations and analytical results fo $p_{c}$

\section{Laplace Transform of the Service and Response Times}

In this section, the Laplace transform of the service time is presented. The unit (or the granularity) of the service time is the period. It is justified by the fact that first the MAC layer can send the next cell only when the current cell has been successfully received by the base station and second by the fact that in this case the client must wait for the next period before sending the next cell. The Laplace transform of the service time is:

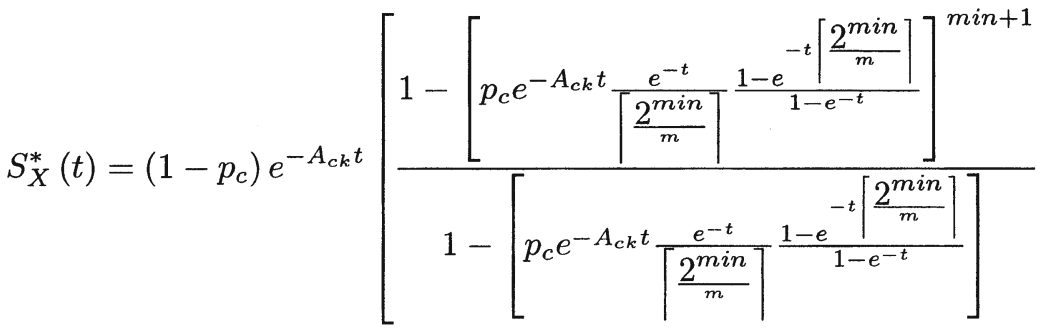

$$
\begin{aligned}
& \left.+\left[\frac{e^{-t}}{\left[\frac{2^{m i n}}{m}\right]} \frac{1-e^{-t}\left[\frac{2^{m i n}}{m}\right\rceil}{1-e^{-t}}\right]\right]^{m i n} \\
& \sum_{n_{r}=m i n+1}^{\max }\left[\left(p_{c} e^{-A_{c k} t}\right)^{n_{r}} \prod_{j=m i n+1}^{n_{r}} \frac{e^{-t}}{\left\lceil\frac{2^{j}}{m}\right\rceil} \frac{\left.1-e^{-t\left\lceil\frac{2^{j}}{m}\right.}\right\rceil}{1-e^{-t}}\right]
\end{aligned}
$$




$$
\begin{aligned}
& +\left[\frac{e^{-t}}{\left[\frac{2^{m i n}}{m}\right]} \frac{\left.1-e^{-t\left\lceil\frac{2^{\min }}{m}\right.}\right\rceil}{1-e^{-t}}\right]^{\min } \prod_{j=m i n+1}^{\max }\left[\frac{e^{-t}}{\left[\frac{2^{j}}{m}\right]} \frac{\left.1-e^{-t\left\lceil\frac{2^{j}}{m}\right.}\right\rceil}{1-e^{-t}}\right]
\end{aligned}
$$

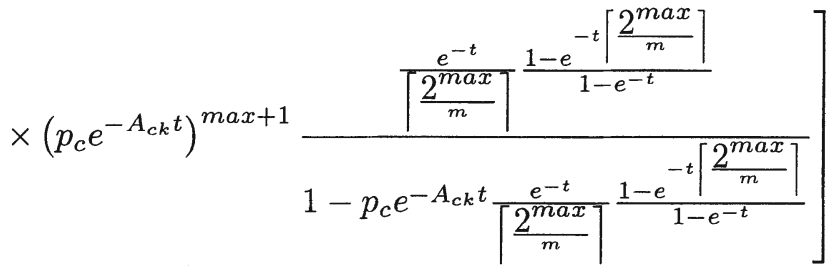

The reader can refer to [6] for details on the calculations and for references about Laplace transform inversion. The response time (because the system under study can be modeled as an $\mathrm{M} / \mathrm{G} / 1$ queue) and the moments of the service time are obtained from the following formulae (where $\rho=\lambda \times \mathbb{E}[X]$ ):

$$
R=\mathbb{E}[X]+\frac{\lambda \mathbb{E}\left[X^{2}\right]}{2(1-\rho)}, \mathbb{E}\left[X^{k}\right]=(-1)^{k}\left(\frac{d^{k} S_{X}^{*}(s)}{d s^{k}}\right)_{s=0}
$$

Since the above Laplace transform has been calculated by using only the first approximation mentionned p.1231, and not the second one, by comparing $\mathbb{E}[X]$ obtained numerically from the Laplace transform with the expression (5), we can validate the second approximation mentionned p.1231.

We can numerically compute as a function of $p_{c}$ the response time by using the derivative (15), or as a function of $\lambda$ by using also the parametrisation given in (9). In fig. 8, the response time is plotted as a function of $\lambda$, for $m=18$, $A_{c k}=3$ and different values for $\min , \max$ and $N$.

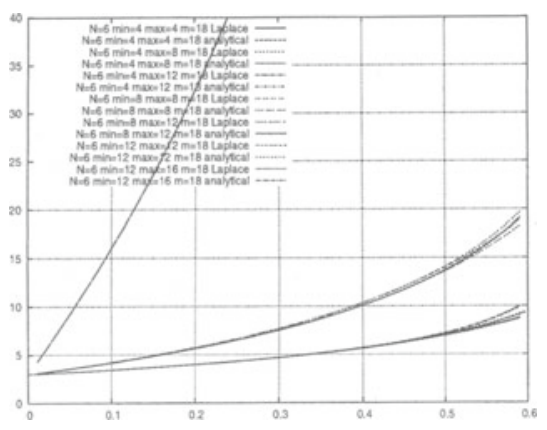

Fig. 7. $\mathbb{E}[X]$, in periods, computed from the Laplace transform and from expression (5), as a function of $p_{c}$

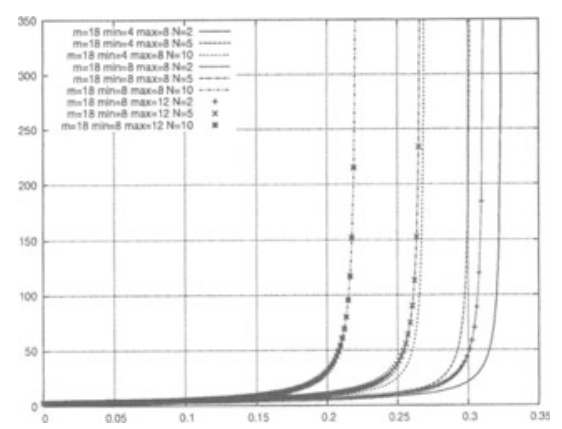

Fig. 8. $R$, in periods, as a function of $\lambda$ 


\section{Performance of the Dynamical Reservation Mechanism}

We assume now that the contention mode is used to transmit dynamical reservation requests. The time is still divided in periods, each period being divided in slots, but there are $m$ slots per period dedicated to reservation requests transmissions and $m^{\prime}$ slots dedicated to data transmisson. When a user wants to send data, he sends one or several messages containing an appropriate number of requests of $m^{\prime}$ data slots by using the contention mechanim. The base station receives the requests, it inserts them in a FIFO queue with constant service time, $m^{\prime}$ slots for a request of $m^{\prime}$ slots, and allocates the required number of slots. The answer to the request is sent to the user who can then send his data by using the allocated data slots. Such a system can be modeled by fig. 9 .

During one period, messages are received during the first $m$ slots and they are served during the last $m^{\prime}$ slots. Let $q_{n}$ be the length of the reservation queue in requests of $m^{\prime}$ slots during the $n^{s t}$ period, $v_{n}$ be the number of arrivals of requests during the $n^{s t}$ period, $\Xi_{i}$ be the number of requests in the $i^{\text {st }}$ message. We assume that all $\Xi_{i}$ are independant and identically distributed (i.i.d.) with a geometric distribution with parameter $\xi$ (denoted in the following $\operatorname{Geo}(\xi)$ ). Let $Q(z)$ be the z-transfom of $q_{n}$ and $V(z)$ the one of $v_{n}$. We assume that the departure process of messages from the users are Poisson with rate $\lambda$. It is a reasonable hypothesis since studies have proved that arrivals of user sessions are Poisson (cf. [7] and [5]).

Moreover, because of the collision recovery algorithm, the sources are not independent, but the message arrival process is a binomial process with parameters $\operatorname{Min}(m, N)$ and $\zeta=\frac{N \lambda}{\operatorname{Min}(m, N)}$, where $\operatorname{Min}(m, N)$ denotes the smallest integer from $m$ and $N$. Actually, at most one arrival can occur per contention slot.

The stationnary limits of $q_{n}$ and $v_{n}$ being $\bar{q}$ and $\bar{v}$, and since $\Xi_{i}$ are independant and $G e o(\xi)$, it can be schown that (see [6]):

$$
Q(z)=\frac{V(z) P(\bar{q}=0)(z-1)}{z-V(z)}, V(z)=\left(\frac{(1-\xi) \zeta}{1-\xi z}+1-\zeta\right)^{M i n(N, m)}
$$

By writing $Q(z)$ under the form $\sum_{i=0}^{+\infty} P(\bar{q}=i) z^{i}$ and we obtain:

$$
\begin{cases}P(\bar{q}=0) & =1-\frac{\xi}{1-\xi} \zeta \operatorname{Min}(N, m) \\ P(\bar{q}=1) & =\left(1-\frac{\xi}{1-\xi} \zeta \operatorname{Min}(N, m)\right)\left(a_{1}-1\right) \\ \forall k \geq 2, P(\bar{q}=k) & =\left(1-\frac{\xi}{1-\xi} \zeta \operatorname{Min}(N, m)\right)\left(a_{k}-a_{k-1}\right)\end{cases}
$$

where, for all $k \geq 1, a_{k}$ is $\left(C_{n}^{p}\right.$ denotes the combination of order $p$ from $n$ elements):

$$
\frac{1}{(1-\zeta)^{\operatorname{Min}(N, m) k}}+\sum_{n=1}^{+\infty} \sum_{l=0}^{k-1}[(1-\xi) \zeta]^{n} \xi^{l} C_{l+n-1}^{n-1} \frac{(-1)^{n} C_{n+\operatorname{Min}(N, m)(k-l)-1}^{\operatorname{Min}(N, m)(k-l)-1}}{(1-\zeta)^{n+\operatorname{Min}(N, m)(k-l)}}
$$


The mean length $L$ of the reservation queue, by differentiating $Q(z)$ and making $z$ going to 1 , and the response time $R^{\prime}$ of the reservation queue (Little's law: $\left.R^{\prime}=L / \Lambda\right)$ are:

$$
L=\frac{1}{P(\bar{q}=0)}\left[1-P(\bar{q}=0)+\operatorname{Min}(N, m) \frac{\zeta \xi^{2}(2-\xi)}{1-\xi}\right], R^{\prime}=\frac{L(1-\xi)}{\lambda N \xi}
$$

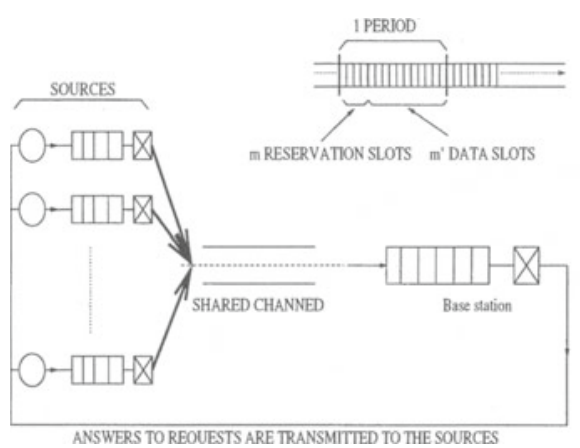

Fig. 9. The reservation model and the way the time is organised

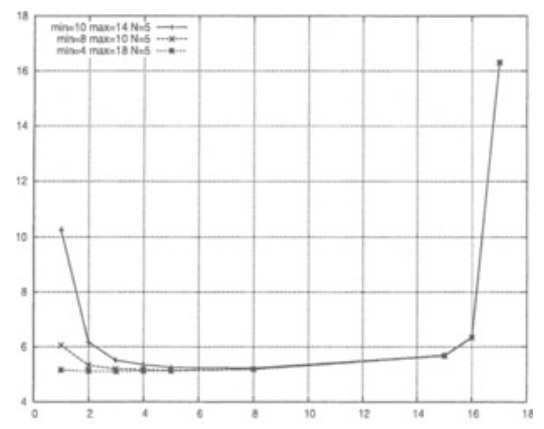

Fig. 10. $R+R^{\prime}$ as a function of $\mathrm{m}$ at a fixed load

The total response time is $R+R^{\prime}$. For a given load, that is when $\lambda$ is fixed and when $\lambda N \frac{\xi}{1-\xi} m^{\prime}=C t$ (where $C t$ is a constant value), and when $\mathrm{m}$ increases but $m+m^{\prime}$ remains constant (i.e.: when the ratio of slots dedicated to send reservation messages over the number of slots dedicated for data transport increases with $m+m^{\prime}$ constant), the number of collisions decreases but the number of requests arriving on the reservation queue is more important compared to the amount of data slots. So, the response time $R$ decreases but $R^{\prime}$ increases: there is a tradeoff to find. Figure 10 presents the total response time as a function of $\mathrm{m}$, for different values for min and max, and with $N=5, \frac{\xi}{1-\xi} m^{\prime}=100$ and $\lambda \simeq 0.0016$ which represents an average load of about 0.8 .

\section{Conclusion}

In this paper, the performance of the European LMDS system is investigated. The characteristic of this system is that it can combine an aloha-kind access mode with a dynamical reservation one. This makes the performance of the system a non-linear function of the parameters of the system. We propose a method to derive the value of the collision rate. The Laplace transform of the service time is also derived. Its inversion remains for future work, but it can already be used to compute numerically the response and service times. At last, the response time of the system when the contention mode is used to send messages for data 
slot reservation is calculated. The distribution of the length of the reservation queue is also analytically computed.

The stability has to be precisely defined. LMDS networks are of course unstable if we consider the expected delay before transmission or the number of messages waiting in the queue. Those two performance criteria tend to infinity when the number of sources increases. But, for a given number of users and a given throughput of the requests of each of them it is interesting to determine the throughput of successfully transmitted packets. Because of max parameter, it appears that when the load increases the successfully transmitted flow do not tend to zero and that is why we say that because of max parameter LMDS networks are stable.

In the future work, the performance of TCP on such a system may be investigated. It seems to be difficult: multi-layer models could be profitably used.

Acknowledgement. This work has been driven within the ERASME project. ERASME is a project funded by the French Réseau National de la Recherche en Télécommunication. The goal of ERASME is to set up an LMDS wireless loop in Limoges (France).

The authors would also like to thank Pr. G. Hébuterne for fruitfull discussions and remarks.

\section{References}

1. D.J. Aldous, Ultimate instability of exponential back-off protocol for acknowledgement-based transmission control of random access communication channels, IEEE Trans. Information Theory, vol. 33, no. 2, 1987.

2. ETSI European Standard (Telecommunications series), Digital Video Broadcasting (DVB); Interaction Channel for Local Multi-point Distribution Systems (LMDS) ETSI EN 301199 V1.2.1 (1999-06).

3. F.P. Kelly, I.M. MacPhee, The number of packets transmitted by collision detect random access schemes, Annals of Probability, vol. 15 pp. 1557-1568, 1987.

4. B.-J. Kwak, N.O. Song, L. E. Miller, Analysis of the Stability and Performance of Exponential Backoff. J. Research of NIST, vol. 108, July-August 2003.

5. Z. Liu, N. Niclausse, C. Jalpa-Villanueva, WAGON: A Web Server Benchmarking Tool. Poster proceedings of the 8th World Wide Web Conference, Toronto, Canada, May 1999.

6. M. Marot, M. Becker, P.-V. Marboua, On the performance of the European LMDS system, Collection des rapports de recherche de l'Institut National des Télécommunications, rapport 03013 RST, 21 octobre 2003.

7. M. Marot, M. Becker, P. Vincent, Modelling a WEB user, application to performance comparisons of UDP versus TCP for WEB traffic transport, 8th International Conference on Telecommunication Systems, 9-12 March 2000, Nashville, Tennessee, USA.

8. R. M. Metcalfe, D. R. Boggs, Ethernet: Distributed packet switching for local computer networks, Communication of the ACM, vol. 19, no. 7, July 1976.

9. P802.11, IEEE standard for wireless lan medium access control (MAC) and physical layer (PHY) specifications, november 1997. 